- That having a hospice-based paracentesis service helped patients to remain in their preferred place of care;

- That our hospice-based paracentesis service significantly decreased the costs to our local acute NHS services.

Method Retrospective review of patient data collected from all ultrasound scans undertaken by the hospice medical team between 2015-2018.

Results Access to ultrasound at the point of care enabled over $95 \%$ of patients to be safely managed within the hospice setting.

Only 3\% of patients required transfer to hospital over a four-year period therefore avoiding either hospital admission or interventional radiology need for paracentesis.

The service was extended to include outpatients and the community. This coincided with an increase in cases with non-malignant liver disease referred to the hospice.

Conclusion The study demonstrated that we are able to avoid hospital admission for the majority of our patients resulting in significant cost savings for our local NHS trust as well as keeping patients in their preferred place of care for symptom management. What are the next steps?

\section{P-172 DRAINING FEELING! RE-AUDIT OF HOSPICE PLEURAL ASPIRATION}

1,2Karen Groves. ' Queenscourt Hospice, Southport, UK; ${ }^{2}$ Edge Hill University, Ormskirk, UK

10.1136/bmispcare-2019-HUKNC.194

Background Hospice pleural aspiration has been undertaken for the last 27 years, following training by a local chest physician. When the requirement for ultrasound (US) guided aspiration was introduced, an ultrasound machine was purchased and staff training undertaken. The British Thoracic Society issued guidance for pleural drainage in 2003 and standards were adapted for use in respect of pleural aspiration in hospice. An audit undertaken in 2015 showed 36 procedures undertaken over a period of three years. 14 of 19 standards were $100 \%$ met; two met in $97 \%$ and two in $94 \%$. Following this there were changes to the documentation template, patient information leaflet and consent form to ensure the capture of missing items.

Method Retrospective review of all pleural aspirations undertaken since the last audit, drawn from electronic clinical record. Documentation of the procedure was audited against 19 standards identified.

Results 16 procedures for eight patients identified. 18 of 19 standards were $100 \%$ met compared to 14 at last audit. In two procedures, aspiration of fluid at the end of instilling local anaesthetic was not confirmed by documentation. One procedure failed to obtain more than $10 \mathrm{ml}$ of fluid despite initial aspiration of fluid via syringe and needle prior to procedure. There were no complications of pneumothorax, pain during procedure, haemorrhage or visceral injury during any of the procedures.

Conclusions Almost all the standards are now comfortably met. Further documentation changes and reminders to clinicians undertaking procedure should ensure thorough documentation. Out of hours procedures should continue to take place when essential for symptom management, but undertaken by most experienced clinician.

\section{Service Development, Models and Collaborative Working}

\section{P-173 THE CARE HOME REVOLUTION - A PERSON CENTRED APPROACH TO EDUCATING CARE HOME STAFF TO DELIVER EOLC}

Rachel Shovelton. Bolton Hospice, Bolton, UK

10.1136/bmjspcare-2019-HUKNC.195

End of life care education in care homes needs to be as person-centred for their staff as the people in their care. Too often care home staff feel undervalued, media attention is frequently negative, and staff receive the brunt of this. Public perception that staff can't do anything else, that they don't really care about people that live in the care home. As a former nursing home manager, now an End of Life Care Educator for care homes, I can provide real examples of support that is tried and tested with care home staff to challenge public perception.

All end of life care education sessions are accessible to all care home staff. Learning needs addressed included literacy, confidence, the fact that some were failed by the education system in our schools. Photos and real stories helps inclusivity, rather than providing handouts. Often staff can recall exact details. Using person-centred approaches during introductions instantly brands sessions as something different to what staff are used to.

An HCA asked if I was the 'new death teacher thingy'. I said I also taught people about living. She stated 'people like her didn't do sessions', 'I'm rubbish at school, at work and no one invites me to these things'. I took this challenge. The visit following my teaching session, I was greeted by the HCA, excited to tell me what she had started to implement, by gut instinct helping staff to identify residents' end of life care needs. She was visibly very proud and so was her manager.

There is lots of negativity in and around care homes. By encouraging support and belief in each other, I am proud to support all care home staff to feel empowered. Care homes are our backbone in the care of our older adults. Where would we be without them? Join the care home revolution.

\section{P-174 SIX STEPS TOO... A UNIQUE, REAL LEARNING AND SUPPORT PROGRAMME OF END OF LIFE CARE FOR CARE HOMES}

Rachel Shovelton. Bolton Hospice, Bolton, UK

\subsection{6/bmjspcare-2019-HUKNC.196}

By using a unique insight as a former nursing home manager and now an End of Life Care educator, I am able to approach the learning needs for all staff with a wide range of techniques. Based in the hospice, I can reflect the good practice of the hospice and demonstrate good practice across all care homes. The programme has been developed using known models such as the Six Steps, but, this is more than that. This is Six Steps Too... The programme has six modules. Each learning session is provided four times across each locality ensuring staff can access them easily (often transport 
is an issue). Every learner completes a knowledge, skills and confidence audit and the results are outstanding, an average increase of $60 \%$ in knowledge, skills and confidence for all learning objectives on every session. Each module is supported by two visits, one for implementation ('show me') one for evidence of the implementation of learning in practice ('prove it').

By using real experiences, the sessions are relevant, true and goals are realistic, often using the mantra ' $I$ can do it so can you', staff soon learn that behind every 'Fred' and 'Doris' (safe names used on each session for confidentiality) is a real person; their legacy is their story, a gift for your learning.

Staff in all care homes I am supporting with the programme have evidence of residents' stories, the impact on all those important to them is enormous.

To conclude, end of life care education for care home staff need not be dull, text book or irrelevant. We need our care home staff to feel motivated and empowered, that is exactly the aim of Six Steps Too...

\section{P-175 HERTS NEIGHBOURS VOLUNTEER NURSING HOME SUPPORT}

Liz Kennedy. Peace Hospice Care, Watford, UK

\subsection{6/bmjspcare-2019-HUKNC.197}

Background Loneliness and social isolation are different but related concepts. Social isolation can lead to loneliness and loneliness can lead to social isolation. They share many factors associated with increasing the likelihood of people experiencing deteriorating health, sensory and mobility impairments (Age UK, 2018).

Following embedding Herts Neighbours volunteer befriending home support, it was identified patients whose permanent place of residence was a nursing home $(\mathrm{NH})$ were not accessing the service.

Aims Uphold our strategic aims and values 'widening access', 'reaching more people'; 'each person gets fair access to care'(Ambitions for palliative and end of life care, National Palliative and End of Life Care Partnership, 2015). Pilot agreed to extend existing model into local nursing home.

Methods February 2018 - Consultation: volunteers and nursing home staff to identify those participating. Volunteer agreement between both organisations. Criteria and consent for pilot inclusion agreed.

June 2018: five patients and three volunteers identified 12 visits made

- Pre- and post- feedback questionnaire;

- Regular support for volunteers from hospice and identified links at nursing home;

- Qualitative feedback from patients, family, staff.

Results Though the sample was small, improvements were identified:

- Loneliness $83 \%$ positive impact;

- Enjoyment of life $83 \%$ positive impact;

- Quality of life 50\% positive impact;

- Specific examples received from family, staff.

Conclusion Evaluation of the pilot showed a positive impact and outcome on quality of life, wellbeing and feelings of loneliness. We learnt that applying the same volunteer coordinator assessment in line with pre-existing process would strengthen the referral and patient feedback completion. Feedback identified actions to improve access and support service. Phase 2 agreed intending to strengthen and reach more people in the future.

Future planning: Consider development of project in line with the outcome of Population Based Needs Assessment report completed 2019.

Project and outcome presented to Internal Governance, Trustee and volunteer groups. Continuing with the project is recommended.

\section{P-176 AN INITIATIVE TO IMPROVE PALLIATIVE CARE PROVISION IN RURAL CARE HOMES}

1,2Suzanne Rainsford, ${ }^{1,2}$ Nicholas Glasgow, ${ }^{2}$ Nikki Johnston. 'Australian National University, Canberra, Australia; ${ }^{2}$ Calvary Health Care - Clare Holland House, Canberra, Australia

\subsection{6/bmjspcare-2019-HUKNC.198}

Background Palliative care in care homes is mostly primary care, with limited access to specialist support, especially in rural settings. To plan for, and recognise anticipated events, thus preventing potentially avoidable hospitalisation and allowing residents to die 'well', requires new approaches to upskill staff. An emerging model, not yet trialled in rural settings, is the introduction of outreach specialist palliative care needs rounds (Chapman, Johnston, Lovell, Forbat et al., 2018; Johnston, Lovell, Liu, Chapman et al., 2019).

Aim To explore, through the experiences of staff and GPs, the provision of end-of-life care within rural care homes prior to, and after the introduction of needs rounds (or case-based educational monthly triage meetings with a palliative medicine specialist [physician] and care home staff).

Design This presentation reports the qualitative inductive data from a larger ethics approved quasi-experimental mixed method study. This ethnographic component utilised 14 open-ended interviews, conducted between 1 March and 18 November 2018, and observational notes. Eleven needs rounds were conducted, commencing 6 April 2018; 25 residents under the care of nine GPs were discussed; 18 care home staff participated in one or more needs rounds. Interviews were audio-recorded, transcribed, and analysed thematically.

Participants: Eight care home staff, working in two facilities, and 3 GPs located in a rural town (population 6,700) in south-eastern NSW, Australia.

Results Participants identified system and site-specific barriers to quality end-of-life care. Needs rounds strengthened: (1) awareness of end of life, reflective practice, and critical thinking by care workers; (2) end-of-life decision making and planning; (3) end-of-life pain management.

Conclusion This study provides a rural perspective on the needs rounds model of care in care homes. While the model of care strengthened the knowledge and confidence of care home staff in providing end-of-life care, its sustainability requires collaboration, co-ordination and increased engagement with GPs. Suggestions for future collaboration through multidisciplinary educational case conferences will be presented. 